Pacific Journal of Mathematic 


\section{RESIDUALLY CENTRAL WREATH PRODUCTS}

\section{ROGER D. KONYNDYK}

This paper is concerned with the problem of determining which standard restricted wreath products of two groups $A$ and $G$ are residually central. Complete characterizations are obtained in the case where $G$ is orderable and in the case where $A$ and $G$ are locally nilpotent.

The contents of this paper formed a part of the author's doctoral dissertation submitted to Michigan State University in 1975. I wish to thank Professor Richard E. Phillips for his guidance and advice. I also wish to thank the referee for his suggestions for simplifying the proofs of Lemma 1, Theorem 2, and Lemma 3.

A group $G$ is said to be residually central if for all $1 \neq x \in G$, $x \notin[x, G]$. Other definitions may be found in [10] and [11]. Residually central groups were first studied by Durbin in [3] and [4]. Further information may be found in papers by Ayoub [1], Slotterbeck [12], and Stanley [13] and [14].

The wreath product of two groups $A$ and $G$ is the semi-direct product $W=\bar{A}] B$, where $\bar{A}$ is the direct sum $\Pi\left\{A_{g} \mid g \in G\right\}$ of copies of $A$. If $\alpha \in \bar{A}$, then $\alpha$ can be written as $\alpha=\prod_{i=1}^{m} a_{i}^{g_{1}}$, meaning that $\alpha\left(g_{i}\right)=a_{i}, 1 \leqq i \leqq m$, and $\alpha(g)=1$ if $g \notin\left\{g_{1}, \cdots, g_{m}\right\}$. If $g \in G$, then $\alpha^{g}=\prod_{i=1}^{m} a_{i}^{g_{i}}$. The subgroup $\bar{A}$ is called the base group of $W$. Note that if $a \in A$, the element $a^{1}$ in $\bar{A}$ can be identified with $a$. Note also that if $B \triangleleft G$, then $(A / B) w r G$ is a homomorphic image of $A w r G$ in the obvious way; the kernel of the homomorphism is $\bar{B}=\Pi\left\{B_{g} \mid g \in\right.$ $G$. Throughout this paper $W$ will denote the wreath product $A w r G$ and $\bar{A}$ its base group.

LEMMA 1. If $g_{1}, \cdots, g_{n} \in G$, then $\prod_{i=1}^{n}\left[g_{i}, G\right]=\left[\left\langle g_{1}, \cdots, g_{n}\right\rangle, G\right]$.

Proof. Since each $\left[g_{\imath}, G\right] \leqq\left[\left\langle g_{1}, \cdots, g_{n}\right\rangle, G\right], \quad \Pi_{i=1}^{n}\left[g_{\imath}, G\right] \leqq$ $\left[\left\langle g_{1}, \cdots, g_{n}\right\rangle, G\right]$. Let $K=\prod_{i=1}^{n}\left[g_{1}, G\right]$, a normal subgroup of $G$. If $Z / K$ is the center of $G / K$, then each $g_{i} \in Z$. Hence $\left\langle g_{1}, \cdots, g_{n}\right\rangle \leqq Z$, and so $\left[\left\langle g_{1}, \cdots, g_{n}\right\rangle, G\right] \leqq K$.

THEOREM 1. Suppose that $W=A w r G$ is residually central. If $G$ is infinite, then $A$ is a $Z$-group.

Proof. Let $a_{1}, \cdots, a_{m} \in A, K=\left\langle a_{1}, \cdots, a_{m}\right\rangle$. By a theorem of Hickin and Phillips [7], it suffices to show that $K \not E[K, A]$. Let $g_{1}, \cdots, g_{m}$ be 
distinct elements of $G$, and set $\alpha=\prod_{i=1}^{m} a_{\imath}^{g_{\imath}} \in \bar{A}$. Since $W$ is residually central, $\quad \alpha \notin[\alpha, W] \geqq[\alpha, \bar{A}]=\prod_{i=1}^{m}\left[a_{i}, A\right]^{g_{1}}$ as a direct sum. Let $b_{i} \in\left[a_{1}, A\right], 1 \leqq i \leqq m$. Then $b_{1}^{g_{1}} \in\left[a_{1}, A\right]^{g_{1}} \leqq[\alpha, W] \triangleleft W$; thus $b_{i}^{1_{G}}=$ $\left(b_{i}^{g_{i}}\right)^{g_{1}^{\prime}} \in[\alpha, W]$. Hence $\prod_{i=1}^{m}\left[a_{i}, A\right]=[K, A] \leqq[\alpha, W] \triangleleft W$, and so $\Pi_{t=1}^{m}\left\{[K, A]^{g} \mid g \in G\right\} \leqq[\alpha, W]$. If $K \leqq[K, A]$, then $a_{\imath} \in[K, A], 1 \leqq i \leqq$ $m$, and $\alpha=\prod_{i=1}^{m} a_{i}^{g_{i}} \in \prod_{i=1}^{m}[K, A]^{g_{i}} \leqq[\alpha, W]$, a contradiction.

Lemma 2. Let $A$ and $G$ be residually central groups. Then $W=$ A $w r G$ is residually central if and only if for all $1 \neq \alpha \in \bar{A}, \alpha \notin$ $[\alpha, G][\alpha, \bar{A}]^{G}$.

Proof. The necessity of the condition follows from the definition of residual centrality.

Let $w \in W$. Since $W$ is a semi-direct product $\bar{A}] G, w$ can be expressed uniquely in the form $\alpha g$, where $\alpha \in \bar{A}$ and $g \in G$. Now $[\alpha g, W] \leqq[\alpha, W][g, \bar{A} G] \leqq \bar{A}[g, G]$. If $g \neq 1$, then $g \notin[g, G]$, since $G$ is residually central. Thus $\alpha g \notin[\alpha g, W]$. If $g=1$, then $[\alpha, W] \leqq$ $[\alpha, G][\alpha, \bar{A}]^{G}$. Hence if $\alpha \notin[\alpha, G][\alpha, \bar{A}]^{G}$, then $W$ is residually central.

A group $G$ is ordered if it possesses a total order $\leqq$ which is preserved under right and left multiplication. Further information may be found in [8]. Orderable groups must be torsion-free. Examples of orderable groups are free groups $[8$, p. 17] and torsion-free locally nilpotent groups [8, p. 16].

THEOREM 2. If $G$ is a residually central orderable group, and $A$ is a $Z$-group, then $W=A w r G$ is residually central.

Proof. Let $\alpha=\prod_{i=1}^{m} a_{i}^{g_{i}} \in \bar{A}$, where $g_{\imath} \in G, a_{\imath} \in A$, and $a_{i} \neq 1,1 \leqq$ $i \leqq m$. By Lemma 2 it is enough to assume that $\alpha \in[\alpha, G][\alpha, \bar{A}]^{G}$ and reach a contradiction. Let $L=\left[\left\langle a_{1}, \cdots, a_{m}\right\rangle, A\right]$. Since $A$ is a $Z$ group, some $a_{\imath} \notin L$, by [7]. If $\bar{L}=\Pi\left\{L^{g} \mid g \in G\right\}$, then $\alpha \notin \bar{L}$, but $\alpha \bar{L} \in \zeta_{1}(\bar{A} / \bar{L})$, where $\zeta_{n}(H)$ denotes the $n$th center of a group $H$. Let $A_{1}=A / L$, and $W_{1}=A_{1} w r G$, a homomorphic image of $W$. Then $\alpha \in[\alpha, W]$ implies that $\alpha \bar{L} \in\left[\alpha \bar{L}, W_{1}\right]$. Because $\alpha \bar{L} \in \zeta_{1}\left(\bar{A}_{1}\right)$, a characteristic subgroup of $\bar{A}_{1},\left[\alpha \bar{L}, W_{1}\right] \leqq \zeta_{1}\left(\bar{A}_{1}\right)$. Let $A_{2}=\zeta_{1}\left(\bar{A}_{1}\right)$; then $W_{2}=$ $A_{2} w r G$ is not residually central, and so we may assume that the base group $\bar{A}$ is abelian. We may also assume that $A=\left\langle a_{1}, \cdots, a_{m}\right\rangle$.

With these assumptions, there is a prime $p$ and subgroup $B$ of index $p$ in $A$. Since some $a_{t} \notin B, \alpha \notin B^{G}$, so that we may factor out $B$ and assume that $A$ is cyclic of prime order $p$. Denoting the field of $p$ elements by $Z_{p}$, we note that $\bar{A}$ is a free $Z_{p} G$-module of rank 1 . Let $\Delta=(1-g \mid g \in G)$ denote the augmentation ideal of $Z_{p} G$. If $g \in G$, then $[\alpha, g]$ may be written in (additive) module notation as $-\alpha+\alpha g=$ 
$-\alpha(1-g)$; thus the assumption that $\alpha \in[\alpha, G]$ means, in module notation, that $\alpha \in \alpha \Delta$. Hence there exists $\delta \in \Delta$ such that $\alpha=$ $\alpha \delta$. Then $\alpha(1-\delta)=0$, and $\alpha \neq 0,1-\delta \neq 0$. But since $G$ is orderable, $Z_{p} G$ can have no zero divisors [10, 26.2 and 26.4], a contradiction.

This shows that if $G$ is a residually central, orderable group, then $A w r G$ is residually central if and only if $A$ is a $Z$-group. For example, free groups are orderable and are residually nilpotent; thus the wreath product of two free groups is residually central.

Lemma 3. Suppose that $W=A w r G$ is residually central, and $G$ has an element $g$ of prime order $p$. Then every element of $A$ and of $G$ of finite order has $p$-power order.

P̈̈oof. Suppose $a \in A$ has prime order $q \neq p$. As elements of $\bar{A}$, $a \neq a^{g}$. However, in a residually central group, elements of relatively prime, finite orders commute [10, Theorem 6.14], and so $a=a^{g}$, which is impossible.

Suppose $h \in G$ has prime order $q \neq p$. Then $g$ and $h$ commute, and $\langle g, h\rangle$ is cyclic of order $p q$. Let $1 \neq a \in A$ and $A_{1}=\langle a\rangle$. Then $W_{1}=A_{1} w r\langle g, h\rangle$ is residually central with an abelian base group. Let $\alpha=[a, g, h]$. Modulo $[\alpha, g]$ we have

$$
1=\left[a, g, h^{q}\right] \equiv[a, g, h]^{q}=\alpha^{q} .
$$

Since $h$ and $g$ commute, and $\bar{A}_{1}$ is abelian,

$$
\begin{aligned}
\alpha=[a, g, h] & =[a, g]^{-1}[a, h]^{-1}[a, g h]=[a, h]^{-1}[a, g]^{-1}[a, h g] \\
& =[a, h, g] .
\end{aligned}
$$

As before, modulo $[\alpha, G]$,

$$
1=\left[a, h, g^{p}\right] \equiv[a, h, g]^{p}=\alpha^{p} .
$$

Thus $\alpha^{p} \in[\alpha, G], \alpha^{q} \in[\alpha, G]$ for the distinct primes $p$ and $q$, so that $\alpha \in[\alpha, G]$, implying that $W$ is not residually central, a contradiction.

Theorem 3. Suppose $A$ and $G$ are locally nilpotent. Then $W=$ A wr $G$ is residually central if and only if either

(1) $G$ is torsion-free, or

(2) For some prime $p$, all elements of $G$ and of $A$ of finite order have p-power order.

Proof. The necessity of (1) or (2) follows from Lemma 3.

If (1) holds, then $G$ is orderable [8, p. 16], and Theorem 2 applies. 
Suppose (2) holds. Since residual centrality is a local property [3], it suffices to show that every finitely generated subgroup $\left\langle w_{1}, \cdots, w_{m}\right\rangle$ of $W$ is contained in a residually central subgroup. Each $w_{1}=\alpha_{1} g_{i}$, where $g_{i} \in G$ and $\alpha_{i} \in \bar{A}$, and each $\alpha_{i}=\prod_{j=1}^{n_{i}} a_{i j}^{g_{i j}}$. Hence

$$
\begin{aligned}
\left\langle w_{1}, \cdots, w_{m}\right\rangle & \leqq\left\langle a_{i j}, g_{l}, g_{\imath} \mid 1 \leqq i \leqq m, i \leqq j \leqq n_{i}\right\rangle \\
& =\left\langle a_{i j}\right\rangle w r\left\langle g_{i j}, g_{\imath}\right\rangle
\end{aligned}
$$

Thus we may assume that both $A$ and $G$ are finitely generated and hence nilpotent.

Let $\alpha=\prod_{k=1}^{l} a_{k}^{g_{k}}$. By Lemma 2, it suffices to assume that $1 \neq \alpha \in$ $[\alpha, G][\alpha, \bar{A}]^{G}$ and reach a contradiction. Since $A$ is nilpotent, there is an integer $r$ such that each $a_{i} \in \zeta_{r}(A)$ and some $a_{i} \notin \zeta_{r-1}(A)$. Then

$$
[\alpha, \bar{A}]^{G} \leqq\left[\left\langle a_{1}, \cdots, a_{l}\right\rangle, A\right]^{G} \leqq\left[\zeta_{r}(A), A\right]^{G} \leqq\left(\zeta_{r-1}(A)\right)^{G} .
$$

$W_{1}=\left(A / \zeta_{r-1}(A)\right) w r G$ is a homomorphic image of $W$ in the obvious way. If $\bar{\alpha}$ denotes the image of $\alpha$ in $W_{1}$, then $\bar{\alpha} \in$ $[\bar{\alpha}, G]\left[\bar{\alpha}, \overline{A / \zeta_{r-1}(A)}\right]=[\bar{\alpha}, G]$ in $W_{1}$, since $\alpha \in[\alpha, G][\alpha, A]^{G}$ in $W$. Let $A_{1}=$ $\zeta_{r}(A) / \zeta_{r-1}(A)$. Thus $A_{1} w r G$ is a subgroup of $W_{1}$ containing $\bar{\alpha} . \quad[\bar{\alpha}, G] \leqq \bar{A}_{1}$, since $A_{1}$ is a characteristic subgroup of $A / \zeta_{r-1}(A)$. By [2, Corollary 2.11], every element of $A_{1}$ of finite order has $p$-power order. By [5, Theorem 2.1], $A_{1}$ and $G$ are residually finite $p$ groups. Because $\bar{\alpha} \in[\bar{\alpha}, G], A_{1} w r G$ is not residually central and therefore not residually nilpotent. Hartley [6], however, has shown that $A_{1} w r G$ is residually nilpotent, a contradiction.

COROllary. If $A$ is abelian and $G$ is locally nilpotent, then $W=A w r G$ is residually central if and only if $W$ is locally a residually nilpotent group.

Proof. The sufficiency of the condition is clear. Theorem 3 and Theorems B1 and B2 of [6] combine to prove the necessity.

\section{REFERENCES}

1. C. Ayoub, On properties possessed by solvable and nilpotent groups, J. Austr. Math. Soc., 9 (1969), 218-227.

2. G. Baumslag, Lecture Notes on Nilpotent Groups, Amer. Math. Soc., (Regional Conference Series in Mathematics, no. 2), Providence, Rhode Island, 1971.

3. J. R. Durbin, Residually central elements in groups, J. Algebra, 9 (1968), 408-413.

4. - On normal factor coverings in groups, J. Algebra, 12 (1969), 191-194.

5. K. W. Gruenberg, Residual properties of infinite solvable groups, Proc. London Math. Soc., 7 (1957), 29-62. 
6. B. Hartley, The residual nilpotence of wreath products, Proc. London Math. Soc., (3) 20 (1970), 365-392.

7. K. K. Hickin, and R. E. Phillips, On classes of groups defined by systems of subgroups, Archiv. der Math., 24 (1973), 346-350.

8. A. I. Kokorin, and V. M. Kopytov, Fully Ordered Groups, transl. D. Louvish., John Wiley and Sons, Inc., New York, 1974.

9. D. S. Passman, Infinite Groups Rings, Marcel Dekker Inc., New York, 1971.

10. D. J. S. Robinson, Finiteness Conditions and Generalized Soluble Groups, Part I, SpringerVerlag, Berlin, 1972.

11. - Finiteness Conditions and Generalized Soluble Groups, Part II, Springer-Verlag, Berlin, 1972.

12. O. Slotterbeck, Finite factor coverings of groups, J. Algebra, 17 (1971), 67-73.

13. T. E. Stanley, Generalizations of the classes of nilpotent and hypercentral groups, Math. Z., 118 (1970), 180-190.

14. - Residual $\mathscr{X}$-centrality in groups, Math. Z., 126 (1972), 1-5.

Received February 18, 1976 and in revised form August 9, 1976.

Ursinus COLlege

Collegeville, PA 19426 




\section{Pacific Journal of Mathematics}

Vol. 68, No. 1

March, 1977

Richard Julian Bagby, On $L^{p}, L^{q}$ multipliers of Fourier transforms . .......

Robert Beauwens and Jean-Jacques Van Binnebeek, Convergence theorems in

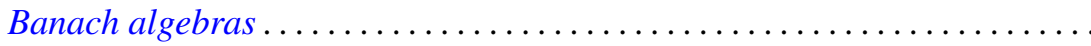

James Cyril Becker, Skew linear vector fields on spheres in the stable

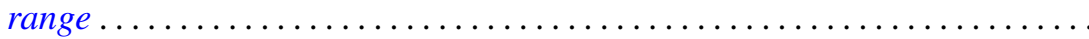

Michael James Beeson, Continuity and comprehension in intuitionistic formal

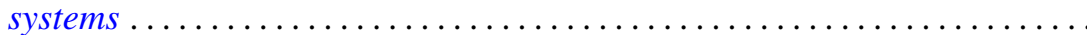

James K. Deveney, Generalized primitive elements for transcendental field

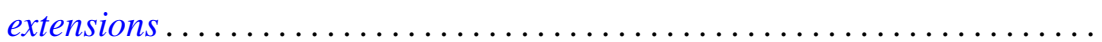

Samuel S. Feder, Samuel Carlos Gitler and K. Y. Lam, Composition properties

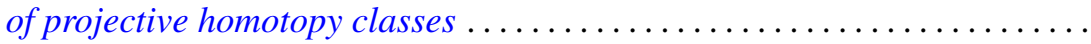

Nathan Jacob Fine, Tensor products of function rings under composition ......

Benno Fuchssteiner, Iterations and fixpoints . . . . . . . . . . . . . .

Wolfgang H. Heil, On punctured balls in manifolds

Shigeru Itoh, A random fixed point theorem for a multivalued contraction

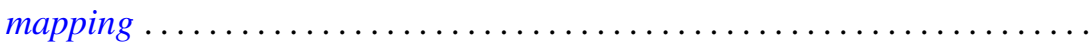

Nicolas P. Jewell, Continuity of module and higher derivations . . . . . . ......

Roger Dale Konyndyk, Residually central wreath products . . . . . . . . . . .

Linda M. Lesniak and John A. Roberts, On Ramsey theory and graphical

parameters.

Vo Thanh Liem, Some cellular subsets of the spheres.

Dieter Lutz, A perturbation theorem for spectral operators

P. H. Maserick, Moments of measures on convex bodies ... . . .

Stephen Joseph McAdam, Unmixed 2-dimensional local domains . .

D. B. McAlister and Norman R. Reilly, E-unitary covers for inverse semigroups...

William H. Meeks, III and Julie Patrusky, Representing codimension-one

homology classes by embedded submanifolds . . .

Premalata Mohapatro, Generalised quasi-Nörlund summability . .

Takahiko Nakazi, Superalgebras of weak-*Dirichlet algebras .

Catherine Louise Olsen, Norms of compact perturbations of operators .

William Henry Ruckle, Absolutely divergent series and isomorphism of

subspaces. II.

Bernard Russo, On the Hausdorff-Young theorem for integral operators .

Arthur Argyle Sagle and J. R. Schumi, Anti-commutative algebras and

homogeneous spaces with multiplications ............

Robert Evert Stong, Stiefel-Whitney classes of manifolds .

D. Suryanarayana, On a theorem of Apostol concerning Möbius functions of

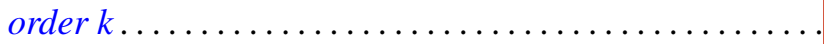

Yoshio Tanaka, On closedness of $C$ - and $C^{*}$-embeddings . . 